\title{
Modeling Film Conductivity for Ion Migration Analysis in Perovskite Solar Cells
}

\author{
NIMA E. GORJI, ${ }^{1,2}$ AMIRHOSEIN MOSAVI, ${ }^{3}$ ARASH ROUHI, ${ }^{4}$ \\ PEZHMAN DARVISHZADEH ${ }^{5}$ and MAHDI GHADIRI ${ }^{6,7,8}$
}

1.- Institute of Research and Development, Duy Tan University, Da Nang 550000, Vietnam. 2.-Faculty of Natural Sciences, Duy Tan University, Da Nang 550000, Vietnam. 3.-Department of Mathematics and Informatics, J. Selye University, Komarno, Slovakia. 4.-Department of New Technologies, University of Tabriz, Tabriz 51566, Iran. 5.-Department of Mechanical Engineering, Shahid Beheshti University, Tehran, Iran. 6.-Informetrics Research Group, Ton Duc Thang University, Ho Chi Minh City, Vietnam. 7.-Faculty of Applied Sciences, Ton Duc Thang University, Ho Chi Minh City, Vietnam. 8.—e-mail: mahdi.ghadiri@tdtu.edu.vn

Ion migration under stress is a major degradation challenge for commercialization of perovskite solar cells. This paper presents three different approaches to model the conductivity variation in perovskite films at elevated temperature, prolonged irradiation levels and strain induced in the lattice. The conductivity variation under any of these stressing conditions can represent ion migration across the perovskite layer because the conductivity is related to mobility and carrier densities. Ion migration can elevate the density of electrons/holes across the film if the stress condition reduce/lift the activation energy at the interface of the film and smoothens the ion passivation to the perovskite layer. In addition, the conductivity of the film is related to device metrics (e.g. fill factor) and also to materials properties (e.g. mobility and bandgap) and, therefore, it can be a significant measure of stress impact on device stability. For example, the conductivity falls under stress which represents the ion migration enhancement. In our modeling, we have shown that the conductivity is inversely related to temperature and strain $(\sigma \propto 1 / k T$, $\sigma \propto 1 / \varepsilon)$ but is directly related to irradiation level $\left(\sigma \propto \xi^{1 / 2}\right)$. We have fitted our modeling to experimental data reported in the literature and extracted the activation energy of ion migration mechanism under every stress condition. The band diagrams of the mechanism are presented and it is shown that stress can foster the ion migration by reducing the activation energy at the interfaces of the perovskite layer. However, the impact of heating is worse on film conductivity, whereas irradiation and strain have a moderate or slight effect on it, respectively. Our findings may provide a practical solution to obtain a measure of ion migration in perovskite solar cells for aging analysis of the cell stability and recovery rates under different stressing conditions.

Key words: Thin films, perovskite, conductivity, strain, temperature, ion migration, degradation

(Received March 19, 2020; accepted May 26, 2020; published online June 5, 2020)

\section{INTRODUCTION}

The power conversion efficiency of organic-inorganic halide perovskite solar cells (PSCs) have rapidly increased to $>23 \%$, but their complex hybrid physico-chemistry is far from being 
understood. ${ }^{1}$ Device instability is currently the main challenge in commercializing perovskite solar cells (PSCs). Apart from perovskite decomposition, ion migration is known to be a serious degradation mechanism for polycrystalline perovskite cells. ${ }^{2}$ The carrier transport in PSCs is yet to be engineered and understood in order to overcome their fast performance degradation rates. Ion migration can cause shunting of the device in another scenario by accelerating migration of metallic ions from the metallic electrode or from the defective area (on the surface of the perovskite layer: $\mathrm{PbI}_{2}$ ) towards the $\mathrm{TiO}_{2} /$ perovskite junction and down to the fluorinedoped tin oxide (FTO) front electrode and creating a short-circuited cell. ${ }^{3,4}$ Several sources of cause or acceleration of ion migration in perovskite and any other polycrystalline material have been identified to be: elevated temperature, prolonged irradiation and lattice strain or a mix of these. ${ }^{5}$ Note that ion migration can even occur due to internal film morphology instabilities under none of these external stress conditions, for example, due to lack of alkali cations $\left(\mathrm{Rb}^{+}, \mathrm{K}^{+}, \mathrm{Na}^{+}\right.$, and $\left.\mathrm{Li}^{+}\right)$or even due to electromigration of $\mathrm{Cu}$ ions at room temperature from the metallic contact towards the conductive oxide electrode across the grain boundaries of the polycrystalline material. ${ }^{6-9}$ Nevertheless, accelerated ion migration will usually occur under a severe or long operation conditions. Zhao et al. ${ }^{10}$ have shown the accelerated ion migration under lattice strain which is created at illumination which causes heat and mismatched thermal expansion between the perovskite and substrate films. Cao et al. ${ }^{11}$ have explained the ion migration through temperaturedependent hysteresis analysis and showed that conductivity can change by ion migration and thus can be a good indicator of ion migration rate. Sveinbjornsson et al. ${ }^{12}$ have measured the impact of prolonged and elevated illumination by photoconductivity measurements on the conductivity of perovskite film. Lee et al. ${ }^{13}$ have performed temperature-dependent conductivity measurements (213-363 K) under dark and optical spectroscopy for different bias conditions and attributed the unusual current-voltage characteristics of the PSCs to ionic migration in halide perovskite materials. It can also be said that the strain can develop by temperature due to mismatch of the thermal coefficients of the adjacent materials in device structure or either due to non-uniform heat during the film fabrication process. Illumination can also heat up the cell. The heat, in turn, causes strain. In all cases, the conductivity shows a change as well. Therefore, we realize that the variation of conductivity by temperature, illumination or strain/stress represents also the ion migration rate and thus can be measured to understand the quality of ion migration mechanism or its acceleration under different stressing conditions.

In this paper, we applied several models of conductivity variation under temperature elevation, irradiation level and strain induced in perovskite film and have correlated the conductivity variation to ion migration across the cell. Our modeling approaches are separately presented for every stressing condition and show the dependence of conductivity individually on irradiation, temperature and mechanical strain in order to explain the experimental data available on these situations. The modeling was compared to the experimental data available in the literature.

\section{THEORY AND MODELING}

Measuring the resistivity or conductivity of the film is a routine practice during the fabrication of solar cells. Many different parameters are interconnected and related to conductivity such as film thickness and mobility. ${ }^{14}$ The conductivity of the thin film is dependent on temperature $(T)$, illumination density $(\xi)$, and mechanical strain $(\varepsilon)$.

\section{Conductivity and Temperature}

Conductivity changes by temperature under the Nernst-Einstein relationship which is given by the Arrhenius relation, ${ }^{10,15}$

$$
\sigma T=\sigma_{0} \exp \left(-\frac{E_{\mathrm{a}}}{k T}\right) \Rightarrow \ln (\sigma T)=-\frac{E_{\mathrm{a}}}{k T}
$$

where $\sigma_{0}$ and $E_{\mathrm{a}}$ are the film conductivity and the activation energy for ion migration, respectively. The slope of the $\ln (\sigma)$ versus $1 / k T$ plot gives the $E_{\mathrm{a}}$. The above equation comes from the conductivity relation to temperature for thermionic emission. A smaller $E_{\mathrm{a}}$ means a smaller potential for ions to pass the barrier which means a faster ion migration rate.

\section{Conductivity and Illumination Intensity}

In general, conductivity is related to electron and hole density and carrier mobility in a semiconductor thin film via, ${ }^{12}$

$$
\sigma=q\left(n \mu_{\mathrm{n}}+p \mu_{\mathrm{p}}\right)
$$

where $\mathrm{n}$ and $\mathrm{p}$ are the electron and hole density in the active layer of the cell and $\mu_{\mathrm{n}}$ and $\mu_{\mathrm{p}}$ are the electron and hole mobility. For a bimolecular recombination, we can get $\mathrm{n}=\mathrm{p}$, thus we can write,

$$
\sigma=q n\left(\mu_{\mathrm{n}}+\mu_{\mathrm{p}}\right)
$$

On the other hand, we know that the recombination rate $(R)$ and the generation rate $(G)$ are given by,

$$
\begin{gathered}
R_{\text {rec }}=B n p \Rightarrow B n^{2} \\
G_{\text {tot }}=\alpha \emptyset_{0} \exp (-\alpha d) \approx A I \xi
\end{gathered}
$$


where $B$ is the recombination rate constant, $A$ is absorbance, and $\xi$ is the illumination intensity. At steady-state conditions under thermal equilibrium the recombination and generation rates are equal,

$$
R=G
$$

An ideal approximation is to assume that all the photons are absorbed and generate electron-hole pairs. Then $G$ is given by,

$$
B n^{2}=A I \xi
$$

From the above equation we get,

$$
n=\sqrt{A I \xi}
$$

Now, if we insert the above equation in Eq. 3 we get,

$$
\sigma=q\left(\mu_{\mathrm{n}}+\mu_{\mathrm{p}}\right) \sqrt{\frac{A I \xi}{B}}
$$

For a mono-molecular recombination the analogy with the above equation reads,

$$
\sigma=q(\mu) \sqrt{\frac{A I \xi}{B}} \Rightarrow \sqrt{\xi}
$$

This is a great result showing that the conductivity changes in the square root function by increasing the illumination level on the film. This has also been shown in Ref. 16 for perovskite solar cells.

\section{Conductivity and Strain}

The conductivity can also change by strain induced under the mechanical stress. Since the conductivity is related to carrier mobility, we can use the deformation potential theory and the effective mass approximation given by, ${ }^{16}$

$$
\mu=\frac{2 q \hbar \Xi}{3 k T\left|m^{*}\right|^{2} E_{g}^{2}}
$$

where $\hbar$ and $m^{*}$ are the reduced Planck constant and effective mass of carriers. $\Xi$ is the elastic modulus and is related to strain $(\varepsilon)$ applied along the transport direction by,

$$
\Xi=\frac{1}{S_{0}} \frac{\partial^{2} E_{\text {tot }}}{\partial \varepsilon^{2}}
$$

where $E_{\text {tot }}$ and $S_{0}$ are the total energy of the thin film layer and the area of its unit cell in equilibrium condition. Several references have shown that the $\Xi$ changes linearly with $\varepsilon{ }^{17-20}$ Therefore, we use the below approximation for the case $\varepsilon$ changes within $1-6 \%$.

$$
\Xi \approx \frac{1}{S_{0} \varepsilon}
$$

Therefore, Eq. 11 changes to below approximation,

$$
\alpha \propto \mu \quad \text { where } \quad \mu=\frac{2 q \hbar^{3}}{3 k T\left|m^{*}\right|^{2} E_{d}^{2} S_{0 \varepsilon}}
$$

where $E_{\mathrm{d}}$ is the deformation potential constant (DPC) along the transport direction and we get it 0.005 which is a very small value and will not impact much on the approximation. Now, by inserting Eq. 14 into Eq. 3 we get the conductivity relation to strain,

$$
\alpha \propto \frac{2 q \hbar^{3}}{3 k T\left|m^{*}\right|^{2} E_{d}^{2} S_{0} \varepsilon} \Rightarrow \alpha \propto \frac{1}{\varepsilon}
$$

this is another practical result meaning that the film conductivity is conversely related to strain.

\section{MODELING RESULTS AND DISCUSSION}

Now we will investigate the application of our models to interpret the practical data available in the literature. The first model considers the variation of perovskite conductivity by temperature. The experimental data were extracted from Zhao et al. ${ }^{10}$ have induced several convex, flat and concave strains into the film and then heated it up to measure the variation of activation energy of the ion migration versus the temperature. Figure 1 shows the fit of the model to these data and Eq. 1 was used to extract the ion migration activation energy of Fig. 2: The schematic of activation energy decreased under strain extracted from Eq. 1 and from conductivity versus temperature. Smaller activation energy means accelerated ion migration. There are

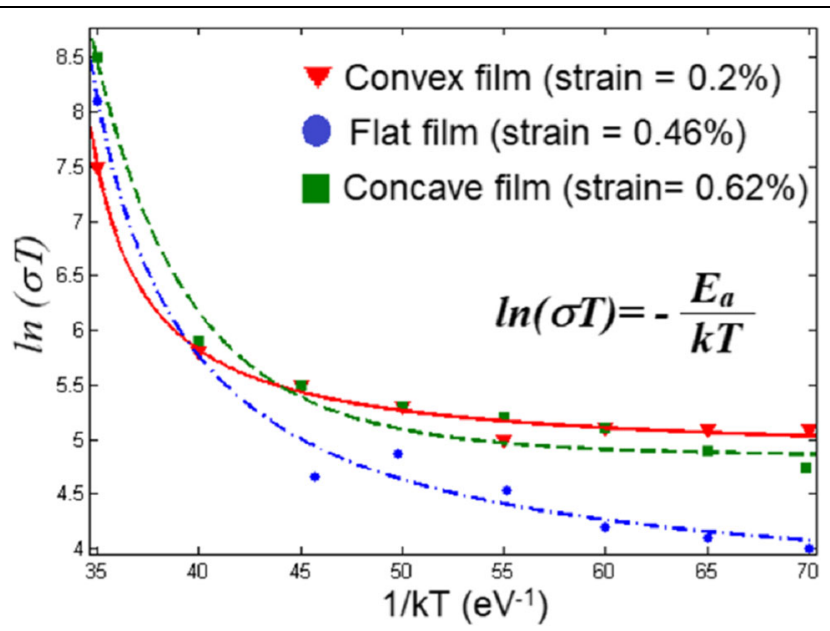

Fig. 1. Variation of perovskite conductivity versus temperature under convex, flat and concave strains. Equation 1 was used to fit with the model and the activation energy extracted. Experimental data were extracted from Ref. 10. 

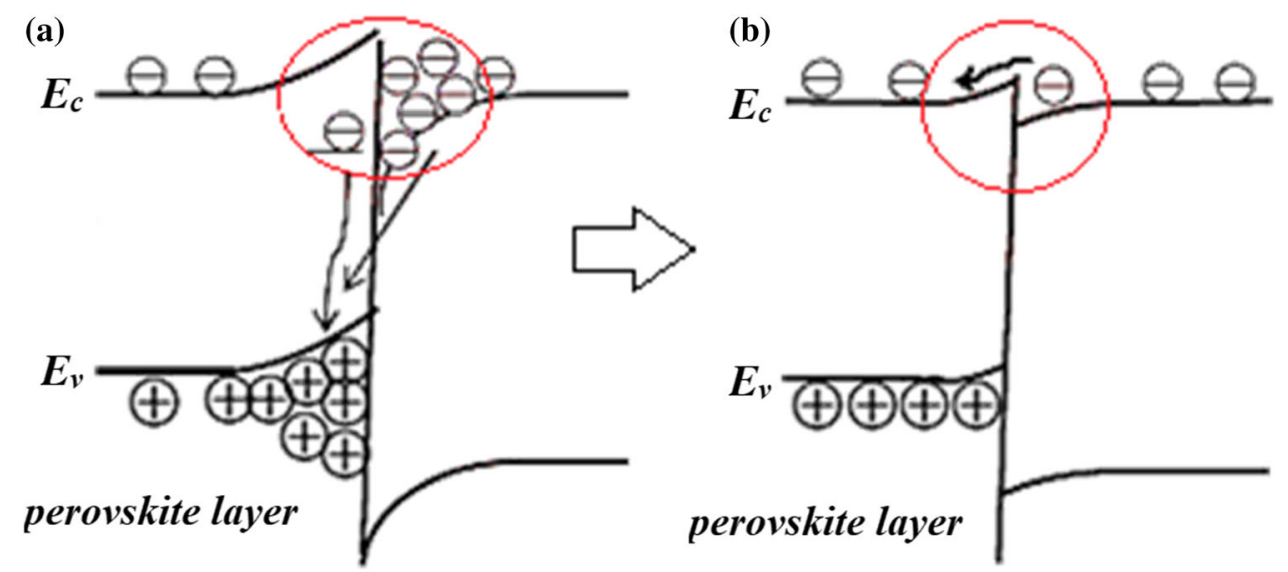

Fig. 2. The schematic of activation energy before (a) and after reducing (b) under strain extracted from Eq. 1 and from conductivity versus temperature. Smaller activation energy in (b) means accelerated ion migration. $E_{\mathrm{c}}$, and $E_{\mathrm{v}}$, refer to conduction and valence band energies, respectively.

three convex, concave and flat strained films in Ref. 10 which obtained comparable results for activation energy of the films from the similar modeling approach. Clearly, a smaller activation energy means a lower potential barrier for carriers to jump into the adjacent layer which is called accelerated ion migration. Ramesh et al. ${ }^{15}$ have also applied the same formula to extract the ion migration activation energy of a ceria-based electrolyte device from the conductivity variation by temperature. For strain = $0.2 \%, 0.47 \%$, and $0.67 \%$ we obtained $E_{\mathrm{a}}=0.29 \mathrm{eV}$, $0.39 \mathrm{eV}$, and $0.53 \mathrm{eV}$ which means a smaller activation energy for higher strain. Note that the three type of strain applied on the film will finally change the lattice strain and finally on potential barrier for the mobile ions and defects. Cao et al. ${ }^{11}$ have reported the activation barriers in the range of $0.06-0.65 \mathrm{eV}$ for a range of temperature variation. These results are in agreement with activation energies reported in Ref. 10.

A strained film under heat will also release or increase its strain. Figure $2 \mathrm{a}$ shows the that the activation energy (spike at the junction) is high and causes the accumulation of carriers at the junction which causes recombination. Figure $2 \mathrm{~b}$ shows the lowered activation energy under temperature or strain and the easy passivation of carriers at the junction which resembles a hampered ion migration. Figure 3 shows the fit of the second modeling for the variation of conductivity under irradiation on two perovskite cells with different $n$-type material: $\mathrm{TiO}_{2}$ and $\mathrm{Al}_{2} \mathrm{O}_{3}$. The experimental data were extracted from Ref. 12. Equation 10 was used to fit with data which is conductively proportional to the square root function of the irradiation increase. It shows that the conductivity increases by irradiation level; however, it also depends on the materials which may impact on mobility for example and thus lower the negative impact of the irradiation on conductivity. One may also discuss that irradiation level also can heat up the cell; therefore, it can be

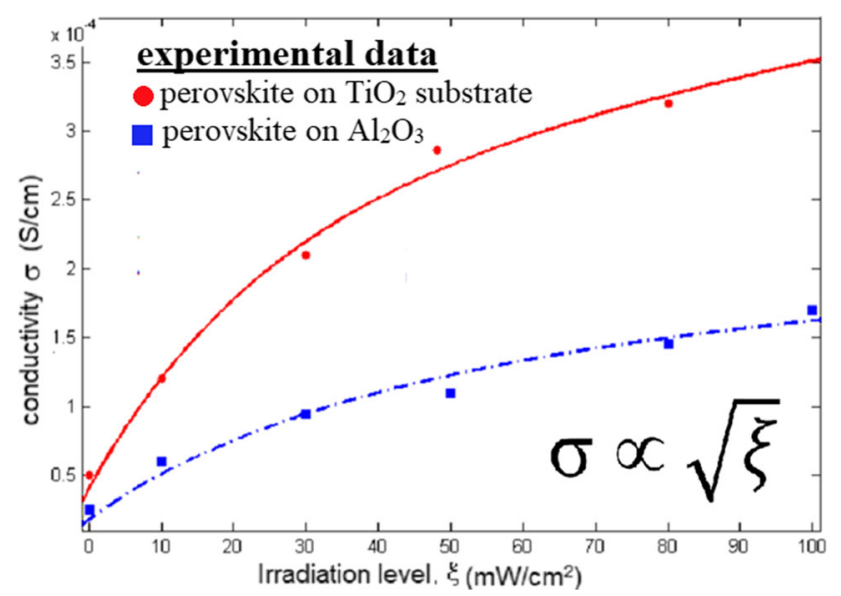

Fig. 3. Variation of conductivity versus irradiation level on perovskite solar cells with $\mathrm{Ti}_{2} \mathrm{O}$ and $\mathrm{Al}_{2} \mathrm{O}_{3}$ substrates. The modeling was fitted with data reported in Ref. 12

considered a heat source which has been modeled in the previous case. However, the irradiation heats up only with infrared wavelength in the spectrum which is only a tiny part of the solar spectra. The square root variation of $\sigma$ versus $\xi$ either is a measure of higher recombination or an accelerated ion migration since the film is getting more conductive for higher $\xi$ which is due to the accelerate metallic (from back contact) ion migration into the perovskite film. Results extracted from this modeling are in agreement with data reported in Ref. 12 .

This migration of mobile ions may be detrimental to cell performance by time if prolonged further. Therefore, a practical measure of our modeling is to plot the conductivity versus irradiation level of the cell to estimate the ion migration rate by illumination level. Ion migration has been shown to be a measure of the degradation of the film under irradiation. ${ }^{19}$ Figure 4 shows the fit of our third modeling approach to the experimental data ${ }^{20}$ 


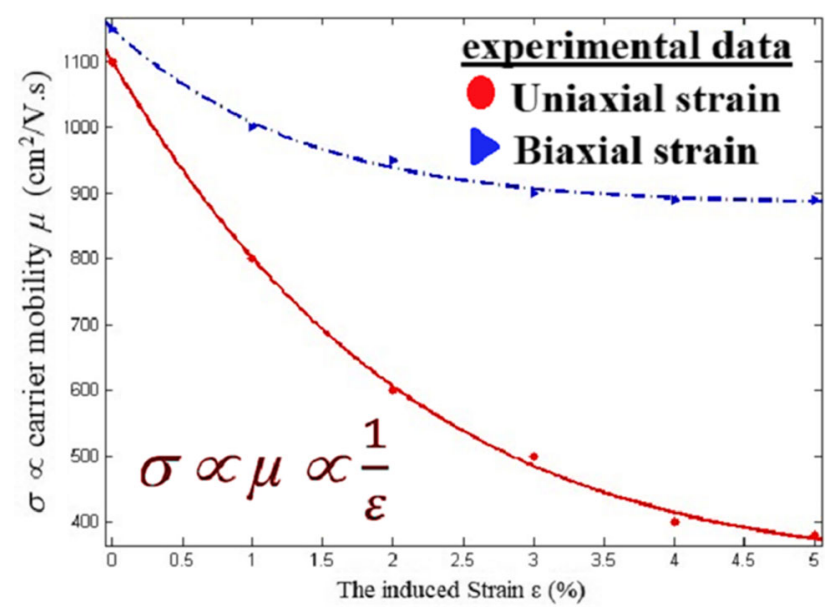

Fig. 4. Variation of conductivity versus the strain level in uniaxial and biaxial directions. The modeling was fitted with data reported in Ref. 20

reported on variation of mobility by strain induced in perovskite solar cells. The Eq. 15 relates the conductivity of the film to the carrier mobility which is a measure of the lattice strain induced in the film. Two different strains were induced to the film: uniaxial strain and biaxial strain. This was reported by Aierken et al. ${ }^{20}$ through theoretical calculations that the strain diminishes the mobility in monolayer $\mathrm{TiS}_{3}$ with a slower slope for biaxial and a steep slope for uniaxial strain. Bennet et al. ${ }^{17}$ have also shown the carrier mobility's dependence on strain in Si-based devices induced by the doping. The significant decrease in mobility at higher strain is due to the creation of more domains which exclusively populates the defects in the lattice and impacts on carrier mobility. ${ }^{18}$ Note that, in this calculation, we have taken the other parameters of Eq. 15 to be constant which is generally a correct assumption since Aierken et al. have shown that by increasing the strain to $6 \%$ at room temperature of $300 \mathrm{~K}$, the $E_{\mathrm{d}}$ and $S_{0}$ will be constant shift in valence band and unit cell area, respectively. Shane et al. ${ }^{7}$ have also reported the activation energy of $0.156 \mathrm{eV}$ for PCBM/TA-PFN, $0.064 \mathrm{eV}$ for PCBM/TA-LiF, and $0.235 \mathrm{eV}$ for PTEG-1 contacted perovskite cells, respectively, which is in good agreement with our results. Strain impact on electrical parameters of the devices has also been investigated in our previous papers in quantum dot based intermediate band solar cells. ${ }^{21}$ Our modeling results are also in good agreement presented in Ref. 19. Another fundamental approach was introduced very recently, where using $\mathrm{Cs}^{+}$based all-inorganic lead halide perovskites is suggested which exhibit much higher stability than $\mathrm{MA}^{+}$cation-based perovskites. ${ }^{22,23}$ Liang et al. ${ }^{23}$ have shown that the all-inorganic perovskite has shown no performance degradation without humidity control even without encapsulation. In another study they also showed that a $\mathrm{CsPb}_{0.9} \mathrm{Sn}_{0.1} \mathrm{IBr}_{2}$ perovskite cell, prepared in ambient atmosphere with a carbon counter electrodes, exhibits good long-term stability and improved endurance against heat and moisture.

\section{CONCLUSION}

Three different modeling approaches were developed to investigate the variation of conductivity by temperature, irradiation level and mechanical strain. It has been shown that conductivity has a converse relationship with temperature and strain and is directly related to irradiation level. The modeling was fitted with experimental data reported in the literature. Conductivity has shown to be a great measure of ion migration kinetics in thin film perovskite solar cells. The activation energy altered due to ion migration is a trend of ion migration mechanism as also presented in the band diagram of Fig. 2. A smaller activation energy means a smaller energy barrier for the carrier passivation at the interface of the perovskite layer which means a faster ion migration rate. Ion migration is a major degradation source in perovskite devices is accelerated under stress conditions or materials decomposition and defect formation. The modeling results presented in this paper are in good agreement with experimental data and the analysis presented in Refs. 10, 12, and 19 The activation energy is close to those calculated in these studies. A practical measure of our findings is that the conductivity can give a measure of stability of degradation rate of the device under a certain stressing condition. Therefore, measuring the conductivity and plotting that against the temperature, strain or irradiation level can disclose the ion migration rate of the film and the possible degradation kinetic.

\section{REFERENCES}

1. https://www.nrel.gov/pv/assets/pdfs/best-research-cellefficie ncies.20191106.pdf.

2. D. Li, F. Sun, C. Liang, and Z. He, J. Alloys Compd. 741, 489 (2018).

3. D.-H. Cho, H.S. Joc, W.J. Lee, T.-G. Kim, B. Shin, S.S. Yoon, and Y.-D. Chung, Sol. Energy Mater. Sol. Cells. 200, 109998 (2019).

4. Y.-H. Kim, C. Wolf, and T.-W. Lee, Nano Energy 2, 329 (2018).

5. T. Zhang, H. Chen, Y. Bai, S. Xiao, and S. Yang, Nano Energy 26, 620 (2016).

6. W. Lee, H. Lic, A.B. Wong, D. Zhang, M. Lai, Y. Yu, Q. Kong, E. Lin, J.J. Urban, J.C. Grossman, and P. Yang, PNAS Proc. 114, 8693 (2017).

7. S. Shao, J. Liu, H.H. Fang, L. Qiu, G.H. Brink, J.C. Hummelen, L.J. Koster, and M.A. Loi, Adv. Energy Mater. 7, 1701305 (2017).

8. C. Wu, L. Jia, S. Guo, S. Han, B. Chi, J. Pu, and L. Jian, ACS Appl. Mater. Interfaces 5, 7886 (2013).

9. R. Chetty, A. Bali, O.E. Femi, and R.C. Mallik, J. Electron. Mater. 45:3, 455 (2016).

10. J. Zhao, Y. Deng, H. Wei, X. Zheng, Y. Shao, J.E. Shield, and J. Huang, Sci. Adv. 3, 5616 (2017).

11. J. Cao, S.X. Tao, P.A. Bobbert, Ch-P Wong, and N. Zhao, Adv. Mater. 30, 1707350 (2018).

12. K. Sveinbjornsson, K. Aitola, X. Zhang, M. Pazoki, A. Hagfeldt, G. Boschloo, and E. Johansson, J. Phys. Chem. Lett. 6, 4259 (2015). 
13. H. Lee, S. Gaiaschi, P. Chapon, D. Tondelier, J.E. Boure, Y. Bonnassieux, V. Derycke, and B. Geffroy, J. Phys. Chem. C 123, 17728 (2019).

14. M. Downs, University of Utah, B.Sc. thesis (2018).

15. S. Ramesh, G. Upender, K.C.J. Raju, G. Padmaja, S.M. Reddy, and C.V. Reddy, J. Mod. Phys. 4, 859 (2013).

16. M. Girtan, Sol. Energy 195, 446 (2020).

17. N.S. Bennett, N. Cowern, and B.J. Sealy, Appl. Phys. Lett. 94, 252109 (2009).

18. B. Jalan, S.J. Allen, G.E. Beltz, P. Moetakef, and S. Stemmer, Appl. Phys. Lett. 98, 132102 (2011).

19. P. Darvishzadeh, Gh Redzwan, R. Ahmadi, and N.E. Gorji, Organ. Electron. 43, 247 (2017).
20. Y. Aierken, D. Çakır, and F.M. Peeters, Phys. Chem. Chem. Phys. 18, 14434 (2016).

21. N.E. Gorji, Sol. Energy 86:3, 935 (2012).

22. J. Liang, C. Wang, Y. Wang, Z. Xu, Z. Lu, Y. Ma, H. Zhu, Y. Hu, C. Xiao, X. Yi, G. Zhu, H. Lv, L. Ma, T. Chen, Z. Tie, Z. Jin, and J. Liu, J. Am. Chem. Soc. 138:49, 15829 (2016).

23. J. Liang, P. Zhao, C. Wang, Y. Wang, Y. Hu, G. Zhu, L. Ma, and J. Liu, J. Am. Chem. Soc. 139:40, 14009 (2017).

Publisher's Note Springer Nature remains neutral with regard to jurisdictional claims in published maps and institutional affiliations. 